\title{
Zn-ZIF EM TECIDO APLICADO NO PROCESSO DE CAPTURA DE $\mathrm{CH}_{4}$
}

\author{
G. A. GIL, G. O. LIMA, L. M. PEDRO, V. H. S. LIMA, F. M. BEZERRA e M. P. MOISÉS \\ ${ }^{1}$ Universidade Tecnológica Federal do Paraná, Departamento de Engenharia Química \\ E-mail para contato: guilherme_gil_@hotmail.com
}

\begin{abstract}
RESUMO - O composto metalorgânico Zn-ZIF-8 foi sintetizado e impregnado em tecido de algodão para aplicação no processo de captura de metano $\left(\mathrm{CH}_{4}\right)$ devido sua capacidade de adsorção e armazenamento de gases. Como o $\mathrm{CH}_{4}$ aparece como um dos principais gases responsáveis pelo efeito estufa, reduzir sua emissão é de fundamental importância no controle dos problemas ambientais. A área específica da Zn-SIF-8 impregnada no tecido foi de $574,3 \mathrm{~m}^{2} \mathrm{~g}^{-1}$ (BET) e área de microporos (t-Plot) de 553,9 $\mathrm{m}^{2} \mathrm{~g}^{-1}$. A fixação do material no tecido foi confirmada por DRX do tecido, da MOF e da MOF impregnada no tecido. Portanto neste trabalho foi avaliado a eficiência da Zn-ZIF em tecido, a $25^{\circ} \mathrm{C}$ e 1 bar, no processo de captura de metano sendo adsorvido $680 \mathrm{~cm}^{3}$ do gás por área $\left(\mathrm{m}^{2}\right)$ do tecido.
\end{abstract}

\section{INTRODUÇÃO}

Segundo Baldé et al. (2016), o potencial de poluição do metano $\left(\mathrm{CH}_{4}\right)$ faz desse gás um dos principais responsáveis pelo efeito estufa. Com isso pesquisadores de diferentes áreas buscam alternativas que visam diminuir a emissão desse gás e consequentemente os impactos ambientais gerados por ele. Os autores Anbia, Hoseini e Sheykhi (2012) apresentam as estruturas metalorgânicas como importante alternativa de controle ambiental devido sua excelente capacidade no processo de adsorção de metano.

Compostos metalorgânicos ou metal-organic frameworks (MOFs), são estruturas cristalinas híbridas porosas, formadas por metais conectados a ligantes orgânicos, que podem ser em uma, duas ou três dimensões (Cliffe et al., 2014). Segundo Wu et al. (2013), as MOFs apresentam grande área específica, volume e uniformidade de diâmetro de poros, além de vários sítios ativos para funcionalização pós-síntese (FPS). De acordo com Rosseinsky (2003), as características estruturais desse material garantem potencial capacidade de adsorver e armazenar gases como, por exemplo, o $\mathrm{CH}_{4}$.

As zeolitic imidazolate frameworks (ZIFs) é um subgrupo das MOFs, que devido sua elevada estabilidade química e térmica é preferencialmente aplicada na captura de gases (Yin et al., 2015). De acordo com Wang et al. (2016), Zn-ZIF são ZIFs que possuem como centro metálico o metal zinco conectado a ligantes orgânicos imidazólicos, e apresentam um microcristal com tamanho médio de $172 \mathrm{~nm}$, volume do microporo de $0,4 \mathrm{~cm}^{3} \mathrm{~g}^{-1}$ e área 
específica de $1200 \mathrm{~cm}^{2} \mathrm{~g}^{-1}$. Os autores Li et al. (2016) ainda afirmam que esta estrutura possui geometria dodecaédrica.

Portanto neste trabalho a Zn-ZIF-8 foi sintetizada utilizando nitrato de zinco e 2metilimidazol com o objetivo de utilizá-la, quando fixada em tecido de algodão, no processo de captura de $\mathrm{CH}_{4}$ a $25^{\circ} \mathrm{C}$, afim de observar sua alta eficiência na adsorção desse gás.

\section{MATERIAIS E MÉTODOS}

\subsection{Síntese do material Zn-ZIF@ tecido}

Para a síntese da estrutura metalorgânica Zn-ZIF-8 foi adicionado 2-metilimidazol em uma solução aquosa de nitrato de zinco. O material foi lavado com água e seco em estufa por 6 horas a $80{ }^{\circ} \mathrm{C}$. Após, foi preparado uma suspensão do material em água $(0,5 \mathrm{~g} / 10 \mathrm{~mL})$ e esta suspensão foi adicionada ao tecido de $100 \%$ algodão $\left(2 \mathrm{~cm}^{2}\right)$. Por último o tecido com o material já em sua superfície foi seco em estufa com ventilação de ar a $60{ }^{\circ} \mathrm{C}$.

\subsection{Caracterização do material Zn-ZIF @ tecido}

A estrutura metalorgânica Zn-ZIF, o tecido de algodão e o material Zn-ZIF@tecido foram caracterizados por difração de raios X (Shimadzu 6000) e fisissorção de $\mathrm{N}_{2}$ a $77 \mathrm{~K}$ (Micromeritics ASAP 2020C). O potencial de adsorção de metano foi avaliado usando 0,5 $\mathrm{m}^{2}$ de tecido no intervalo de pressão entre 0,01 e 1 bar.

\section{RESULTADOS E DISCUSSÃO}

Foi feita a caracterização das propriedades texturais da Zn-ZIF-8, assim como sua área específica total e de seus microporos, por meio da fisissorção de $\mathrm{N}_{2}$ (a), o DRX do tecido, da MOF e do tecido de algodão já com a MOF impregnada em sua superfície (b), a isoterma de adsorção/dessorção de $\mathrm{CH}_{4}$ no tecido impregnado com MOF a $30{ }^{\circ} \mathrm{C}$ (c) e adsorção de $\mathrm{CH}_{4}$ utilizando o tecido com MOF afim de determinar o volume de gás adsorvido por metro quadrado de tecido, conforme mostra a Figura 1.

Na Figura 1(a) é apresentada a isoterma de fisissorção de $\mathrm{N}_{2}$ a $77 \mathrm{~K}$ para a Zn-ZIF-8. Esta isoterma é classificada segundo a IUPAC como do tipo I, característica de sólidos microporosos. A área específica calculada pelo método BET foi de $574,31 \mathrm{~m}^{2} \mathrm{~g}^{-1}$ e área de microporos (t-Plot) de 553,8936 $\mathrm{m}^{2} \mathrm{~g}^{-1}$. Estas propriedades texturais estão de acordo com os resultados reportados na literatura, como exemplo, Barbosa et.al., que encontrou área de 843 $\mathrm{m}^{2} \mathrm{~g}^{-1}$ para a Zn-ZIF-8 sintetizada com razão molar Zn:IM 1:4. Vale a pena destacar que a área específica apresentada pelo material ZIF@ tecido é extremamente relevante e inovadora, visto que esta medida é representada pela relação área/massa do material e no material ZIF@tecido a massa é maior do que nos materiais Zn-ZIF-8 reportados na literatura, pois considera-se a massa do tecido, subestimando a área da Zn-ZIF-8. A formação da estrutura cristalina Zn-ZIF e sua fixação no tecido pode ser confirmada na Figura 1(b) por comparação entre os 
difratogramas de raios $\mathrm{X}$ da amostra da MOF sintetizada com o do tecido com MOF impregnada, onde os sinais de difração se sobrepõem, indicando que há Zn-ZIF no tecido. Os planos cristalinos característicos da estrutura ZIF encontrados estão de acordo com o padrão reportado na literatura (Yo et. al., 2015). Na Figura 2(c), são mostrados os resultados de adsorção de $\mathrm{CH}_{4}$, obtendo o volume adsorvido deste gás por massa do material Zn-ZIF, chegando a um valor de $3,4 \mathrm{~cm}^{3} \mathrm{~g}^{-1}$, quando a pressão é de 1,0 bar. Enquanto que na Figura 1(d) relaciona o volume adsorvido de $\mathrm{CH}_{4}$ com a área $\left(\mathrm{m}^{2}\right)$ de tecido, obtendo o valor de 680 $\mathrm{cm}^{3} \mathrm{~m}^{-2}$, também a 1,0 bar.

Figura 1 - Representação das propriedades texturais (a). DRX do tecido, da MOF e do tecido impregnado com MOF (b). Isoterma de adsorção/dessorção de $\mathrm{CH}_{4}$ (c). Adsorção de $\mathrm{CH}_{4}$ usando o tecido impregnado com MOF, destacando o volume de metano adsorvido por $\mathrm{m}^{2}$ de tecido $(\mathrm{d})$.


Portanto, o material desenvolvido neste trabalho pode ser aplicado ao processo de captura de $\mathrm{CH}_{4}$ da atmosfera quando impregnado em um tecido. Este fato é comprovado observando a quantidade adsorvida do gás analisado em pressões relativas entre 0 (vácuo) e 1,0 bar.

\section{CONCLUSÃO}

Com o avanço nas diferentes áreas da ciência e com o surgimento de materiais como as MOFs, criam-se novas maneiras de solucionar os problemas ambientais que tanto afetam o 
planeta Terra, como por exemplo a emissão de gás metano. Pelos resultados apresentados no presente trabalho, observou-se que a Zn-ZIF-8 em tecido de algodão apresenta potencial capacidade de aplicação no processo de adsorção de $\mathrm{CH}_{4}$ fazendo desse material uma excelente alternativa no controle ambiental.

\section{REFERÊNCIAS}

ANBIA, M.; HOSEINI, V.; SHEYKHI, S.. Sorption of methane, hydrogen and carbon dioxide on metal-organic framework, iron terephthalate. Journal of Industrial and Engineering Chemistry, p. 1149-1152, 2012.

BAldÉ, H.; VANDERZAAG, A. C.; BURTT, S. D.; RIDDLE, C. W.; CROLlA, A.; DESJARDINS, R. L.; MACDONALD, D. J.. Methane emissions from digestate at an agricultural biogas plant. Bioresource Technology, p. 914-922, 2016.

BARBOSA, P; ROSERO-NAVARRO, N. C.; SHI, F.; FIGUEIREDO, F. M. L. Protonic Conductivity of Nanocrystalline Zeolitic Imidazolate Framework 8. Eletrochimica Acta, 2015, 19-27.

CLIFFE, M. J.; WAN, W.; ZOU, X.; $\quad$ CHATER, P. A.; KLEPPE, A. K.; TUCKER, M. G.; WILHELM, H.; FUNNELL, N. P.; COUDERT, F. X.; GOODWIN, A. L.. Nature Communications, 2014, 5 (4176), 1-9.

LI, Y.; ZHOU, K.; HE, M.; YAO, J.. Synthesis of ZIF-8 and ZIF-67 using mixed-base and their dye adsorption. Microporous and Mesoporous Materials, p. 287-292, 2016.

ROSSEINSKY, M. J.. Recent developments in metal-organic framework chemistry: design, discovery, permanent porosity and flexibility. Microporous and Mesoporous Materials, p. 1530,2003

YIN, H.; LEE, T.; CHOI, J.; YIP, A. C. K.. On the zeolitic imidazolate framework-8 (ZIF-8) membrane for hydrogen separation from simulated biomass-derived syngas. Microporous and mesoporous materials.

YU, H.; QIU, X.; NEELAKANDA, P.; DENG, L.; KHASHAB, N.; NUNES, S.; PEINEMANN, K.. Hollow ZIF-8 Nanoworms from Block Copolymer Templates. Sci. Rep. 5, 15275; doi: 10.1038/srep15275, 2015

WANG, C.; YANG, F.; SHENG, L.; YU, J.; YAO, K.; ZHANG, L.; PAN, Y.. Zincsubstituted ZIF-67 nanocrystals and polycrystalline membranes for propylene/propane separation. Chem. Communications, p. 12578-12581, 2016

WU, H.; CHUA, I. S.; KRUNGLEVICIUTE, V.; TYAGI, M.; CHEN, P.; YILDIRIM, T.; ZHOU, W.. J. Am. Chem. Soc. 135, 10525-10532, 2013 